\title{
Ceric ammonium nitrate (CAN) catalyzed one-pot synthesis of fully substituted new indeno[1,2-b]pyridines at room temperature by a multi-component reaction
}

\author{
Pradip Kumar Tapaswi and Chhanda Mukhopadhyay* \\ Department of Chemistry, University of Calcutta, 92 APC Road, Kolkata 700 009, India \\ E-mail: cmukhop@yahoo.co.in
}

\begin{abstract}
Ceric ammonium nitrate (CAN) $(10 \mathrm{~mol} \%)$ in ethanol has been found to be an efficient catalyst for the one-pot multi-component synthesis of fully substituted new indeno[1,2- $b]$ pyridines through a combination of 1,3-indandione, propiophenone or 2-phenylacetophenone, aromatic aldehydes, and ammonium acetate at room temperature. The same methodology also worked well for the construction of several other 2,3,4,5,6-pentasubstituted pyridines at room temperature. Atom economy, excellent yields and mild reaction conditions are some of the important features of this protocol.
\end{abstract}

Keywords: Ceric ammonium nitrate (CAN), indeno[1,2-b]pyridines, room temperature, 2,3,4,5,6-pentasubstituted pyridines

\section{Introduction}

The development of new and efficient synthetic methodologies for the rapid construction of potentially bio-active compounds constitutes a major challenge for chemists in organic synthesis. Many biologically important molecular scaffolds can easily be synthesized from readily available starting materials with the help of the multi-component reactions (MCRs). ${ }^{1}$ MCRs allow the construction of several bonds in a single operation and are getting considerable importance as one of the most powerful emerging synthetic tools for the creation of molecular complexity and diversity. ${ }^{2}$

The indenopyridine nucleus is present in the 4-azafluorenone group of alkaloids, represented by its simplest member onychnine. ${ }^{3}$ Many indenopyridine derivatives were found to exhibit adenosine A2a receptor binding and phosphodiesterase inhibiting activities for the treatment of neurodegenerative disorders and inflammation related diseases. ${ }^{4}$ They also behave as calcium antagonistic activators, ${ }^{5}$ and herbicides. ${ }^{6}$ Therefore, these compounds have recognized themselves as heterocycles of significant chemical and biological importance. As a result, the 
ready and efficient synthesis of these molecules has attracted considerable attention from the synthetic organic community.

Several methods are available describing the synthesis of the $5 H$-indeno[1,2- $b$ ]pyridin-5ones: reaction of the Knoevenegal products of several 1,3-indandiones and aromatic aldehydes with some substituted cyanoacetamide (or phenyl acetonitrile or ethyl phenylacetate and ammonium acetate), ${ }^{7}$ oxidative thermal rearrangement of 2 -indanone oxime $O$-allyl ethers, ${ }^{8}$ direct cyclization of 2-aryl-3-methylpyridines to give $5 H$-indeno[1,2- $b$ ]pyridines followed by oxidation, ${ }^{9}$ cyclization of 2-aryl-3-nicotinic acids by the use of polyphosphoric acid, ${ }^{9,10}$ Pummerer reaction of imidosulfoxides, ${ }^{11} \mathrm{Pd}(0)$-catalyzed cross-coupling reaction ${ }^{12}$ between arylboronic acids and 2-halopyridines. Tu et al. developed the synthesis of substituted indeno[1,2- $b$ ]pyridines by the multi-component reaction of aldehydes, 1,3-indandione or 1indenone and aromatic ketones in the presence of ammonium acetate under microwaveirradiation. ${ }^{13}$ Most of these methodologies suffer from one or more serious drawbacks such as laborious and complex work-up and purification, strong basic and acidic conditions, multistep reactions and occurrence of side reactions, low yields, and the use of expensive reagents. In addition, most of the earlier-reported methodologies require elevated temperature created by either microwave-oven irradiation or heating the reaction mixture at high temperature. Very recently, we have reported the synthesis of several indeno[1,2-b]pyridine derivatives by the multi-component reaction of 1,3-indandione, aldehydes, 2-acetylthiophene or 2-acetylfluorene and ammonium acetate at room temperature using L-proline $(15 \mathrm{~mol} \%)$ as catalyst. ${ }^{14}$ However, in that case the 3-position of the pyridine ring system was unoccupied and still there is only one report in this regard. ${ }^{7}$

Recently, ceric ammonium nitrate (CAN) received considerable attention as an inexpensive, nontoxic and readily available catalyst for the construction of carbon-carbon and carbonheteroatom bonds, ${ }^{15}$ due to its excellent solubility in water, cost-effectiveness, eco-friendly nature, easy handling, high reactivity and easy work-up procedures. In addition, CAN is able to catalyze various organic transformations not only based on its electron transfer capacity but also with its Lewis acidic property. ${ }^{16} \mathrm{~J}$. R. Pfister reported the oxidation of Hantzsch-type 1,4dihydropyridines using two equivalents of ceric ammonium nitrate (CAN). ${ }^{17} \mathrm{CAN}$ has also been used as an active catalyst for the construction of substituted 6-alkoxy-2-methyl-1,4,5,6tetrahydropyridines by a four-component reaction between primary aliphatic amines, $\beta$ ketoesters or $\beta$-ketothioesters, $\alpha, \beta$-unsaturated aldehydes and alcohols. ${ }^{18}$ Menéndez et al. reported the synthesis of 1-allyl(propargyl)-6-allyl(propargyl)oxy-1,4,5,6-tetrahydropyridines by a CAN catalyzed multi-component reaction (MCR). ${ }^{19}$ Excellent catalytic activity of CAN has also been manifested in the synthesis of 3-amino-4-aryl-4-hydroxypiperidines, ${ }^{20}$ polyhydroquinoline derivatives, ${ }^{21}$ and $N$ - substituted decahydroacridine-1,8-dione derivatives. ${ }^{22}$ 


\section{Results and Discussions}

In continuation of our synthesis towards biologically important heterocycles, ${ }^{23}$ we report herein, a simple, mild and efficient synthesis of densely substituted indeno[1,2- $b$ ]pyridines by the multicomponent reaction of 1,3-indandione, aldehydes, propiophenone or 2-phenylacetophenone and ammonium acetate in excellent yields employing ceric ammonium nitrate (CAN) $(10 \mathrm{~mol} \%)$ as a catalyst at room temperature $\left(25-30{ }^{\circ} \mathrm{C}\right)($ Scheme 1$)$.

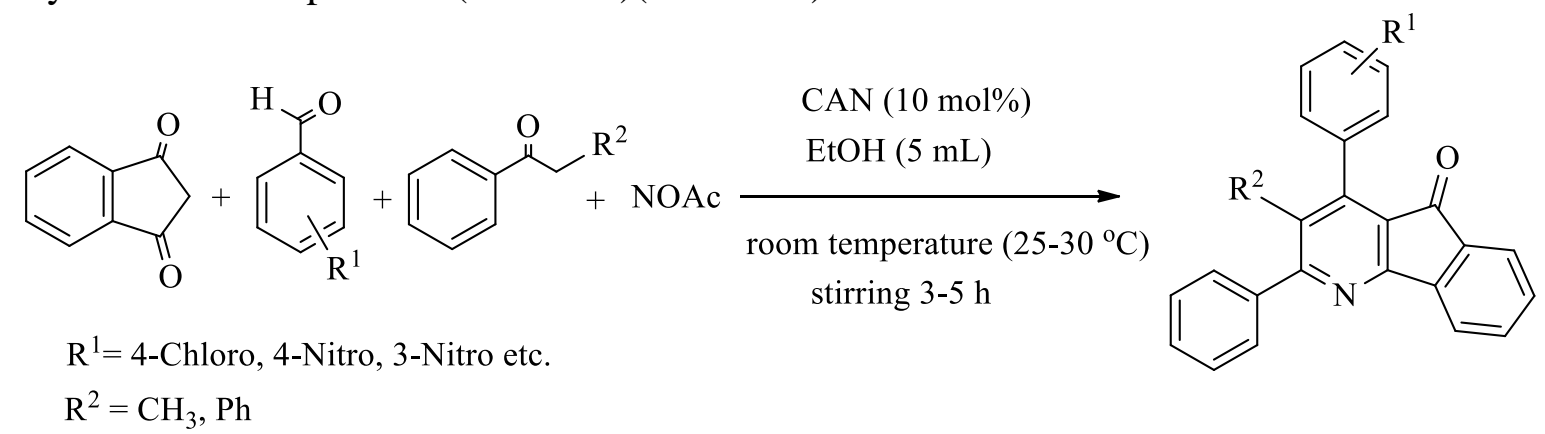

Scheme 1. Synthesis of fully substituted indenopyridine at room temperature $\left(25-30{ }^{\circ} \mathrm{C}\right)$.

Initially, we explored the four component reaction of 1,3-indandione (1 mmol), propiophenone $(1 \mathrm{mmol})$, 4-nitrobenzaldehyde $(1 \mathrm{mmol})$ and ammonium acetate $(1.3 \mathrm{mmol})$. All these four components were first mixed and stirred at room temperature $\left(25-30{ }^{\circ} \mathrm{C}\right)$ in ethanol $(5$ $\mathrm{mL}$ ) which resulted in isolation of only $30 \%$ of the desired product. To enhance the yields and reduce the reaction time, several metal catalysts were examined. Using $\mathrm{FeCl}_{3} .6 \mathrm{H}_{2} \mathrm{O}(15 \mathrm{~mol} \%)$ as a catalyst, the reaction proceeded smoothly to afford the desired product in good yields (75\%, Table 1, entry 3). On the other hand, $\mathrm{InCl}_{3}, \mathrm{RuCl}_{3}$ and $\mathrm{VO}(\mathrm{acac})_{2}$ gave intermediate yields (Table 1, entries 5-7). Surprisingly, using ceric ammonium nitrate (CAN) (10 mol\%, Table 1, entry 9) as catalyst in ethanol best result both in terms of yields and reaction time was obtained. Ethanol (5 $\mathrm{mL}$ ) proved to be a much better solvent in terms of yields than all the other tested solvents including $\mathrm{CH}_{2} \mathrm{Cl}_{2}$, THF, MeCN and even $\mathrm{H}_{2} \mathrm{O}$ (Table 1, entries 10-13) which afforded the desired products in moderate yields. Therefore, the optimized conditions for the indeno[1,2$b]$ pyridines synthesis consist of the combination of 1,3-indandione (1 mmol), propiophenone (1 mmol), benzaldehydes ( $1 \mathrm{mmol})$, ammonium acetate $(1.3 \mathrm{mmol})$, ceric ammonium nitrate (CAN) $(10 \mathrm{~mol} \%)$ and $\mathrm{EtOH}(5 \mathrm{~mL})$ at room temperature $\left(25-30{ }^{\circ} \mathrm{C}\right)$ (Table 1, entry 9$)$. 
Table 1. Synthesis of densely substituted indenopyridine at room temperature $\left(25-30{ }^{\circ} \mathrm{C}\right)$

\begin{tabular}{ccccc}
\hline Entry & Catalyst (mol\%) & $\begin{array}{c}\text { Solvents } \\
(\mathrm{mL})\end{array}$ & Time (h) & Yields (\%) \\
\hline 1 & - & $\mathrm{EtOH}(5)$ & 24 & 30 \\
2 & $\mathrm{FeCl}_{3}, 6 \mathrm{H}_{2} \mathrm{O}(10)$ & $\mathrm{EtOH}(5)$ & 12 & 70 \\
3 & $\mathrm{FeCl}_{3}, 6 \mathrm{H}_{2} \mathrm{O}(15)$ & $\mathrm{EtOH}(5)$ & 12 & 75 \\
4 & $\mathrm{FeCl}_{3}, 6 \mathrm{H}_{2} \mathrm{O}(20)$ & $\mathrm{EtOH}(5)$ & 12 & 75 \\
5 & $\mathrm{InCl}_{3}(15)$ & $\mathrm{EtOH}(5)$ & 12 & 65 \\
6 & $\mathrm{RuCl}_{3}(15)$ & $\mathrm{EtOH}(5)$ & 14 & 48 \\
7 & $\left.\mathrm{VO}_{2} \mathrm{acac}\right)_{2}(15)$ & $\mathrm{EtOH}(5)$ & 15 & 45 \\
8 & $\left(\mathrm{NH}_{4}\right)_{2} \mathrm{Ce}\left(\mathrm{NO}_{3}\right)_{6}(15)$ & $\mathrm{EtOH}(5)$ & 4.5 & 94 \\
9 & $\left(\mathrm{NH}_{4}\right)_{2} \mathrm{Ce}\left(\mathrm{NO}_{3}\right)_{6}(10)$ & $\mathrm{EtOH}(5)$ & 4.5 & 94 \\
10 & $\left(\mathrm{NH}_{4}\right)_{2} \mathrm{Ce}\left(\mathrm{NO}_{3}\right)_{6}(10)$ & $\mathrm{CH}{ }_{2} \mathrm{Cl}(5)$ & 5 & 52 \\
11 & $\left(\mathrm{NH}_{4}\right)_{2} \mathrm{Ce}\left(\mathrm{NO}_{3}\right)_{6}(10)$ & $\mathrm{THF}(5)$ & 5 & 45 \\
12 & $\left(\mathrm{NH}_{4}\right)_{2} \mathrm{Ce}\left(\mathrm{NO}_{3}\right)_{6}(10)$ & $\mathrm{MeCN}(5)_{2}$ & 5 & 65 \\
13 & $\left(\mathrm{NH}_{4}\right)_{2} \mathrm{Ce}\left(\mathrm{NO}_{3}\right)_{6}(10)$ & $\mathrm{H}_{2} \mathrm{O}(5)$ & 24 & 20 \\
\hline
\end{tabular}

Once the optimized condition for the indeno[1,2-b]pyridines synthesis was achieved, several aromatic aldehydes possessing both electron donating and electron withdrawing groups were tested (Table 2) under the same reaction condition which revealed that the reaction gave higher yields of indeno[1,2- $b$ ]pyridines when the aromatic aldehydes bear an electron-withdrawing substituents probably because the Michael addition is easier. The reaction was also examined with 2-phenylacetophenone in place of propiophenone in very good yields of the products (Table 2, entries 7-11).

Table 2. Synthesis of densely substituted indenopyridine at room temperature $\left(25-30{ }^{\circ} \mathrm{C}\right)$

Entry Products $\quad$ Time (h) $\begin{gathered}\text { Yields (\%) } \\ \text { (isolated) }\end{gathered}$


Table 2. Continued

Time (h) $\begin{gathered}\begin{array}{c}\text { Yields (\%) } \\ \text { (isolated) }\end{array} \\ \text { Entry }\end{gathered}$


Table 2. Continued

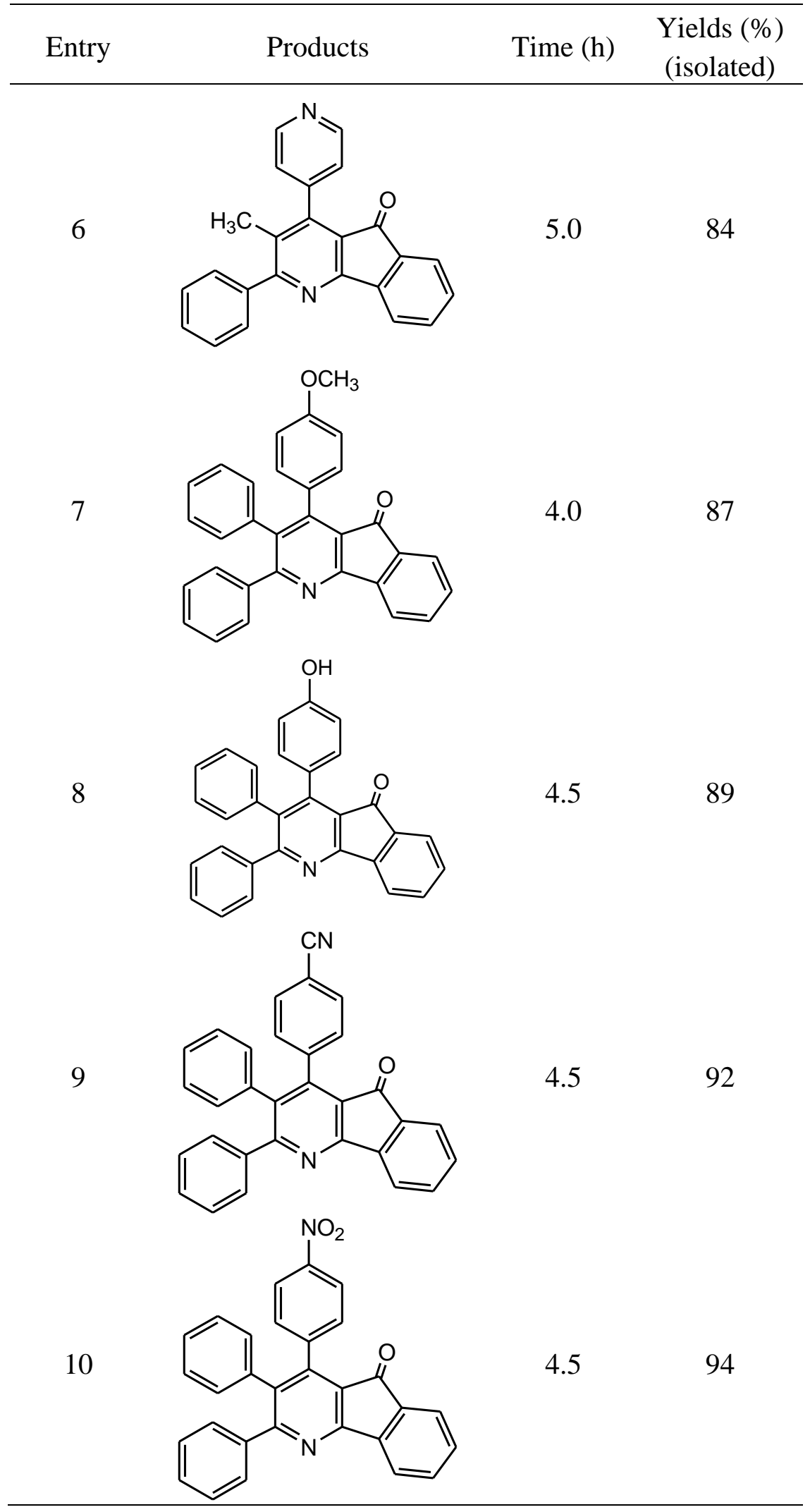


In all cases, the crude products obtained by extracting the reaction mixture with ethyl acetate (EtOAc) were purified by crystallization from hot ethanol. All the final products were characterized by IR, ${ }^{1} \mathrm{H}$ NMR and elemental analyses. The structure of one of the products (Table 2, entry 10) has been confirmed by X-ray crystal structure analysis of its single crystal and is shown below in Figure 1.

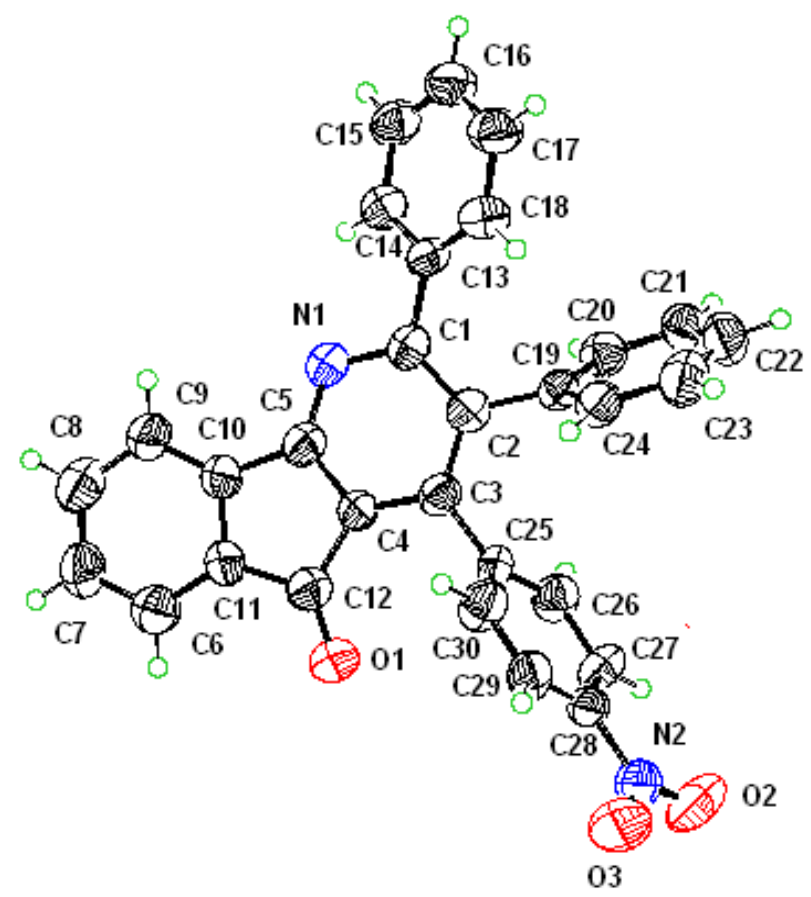

Figure 1. ORTEP diagram of a single crystal of 2,3-Diphenyl-4-(4-nitrophenyl)-indeno[1,2b] pyridin-5-one (Table 2, entry 10) showing the crystallographic numbering (CCDC 811809).

A probable mechanism for ceric ammonium nitrate (CAN) catalysed synthesis of indeno[1,2$b$ ]pyridines has been proposed (Scheme 2) in which the reaction proceeds through the activation of aldehydic carbonyl oxygen by ceric ammonium nitrate (CAN) by virtue of its inherent Bronsted acidity which makes it capable of bonding with the carbonyl oxygen increasing the reactivity of the parent aldehyde carbonyl and subsequent condensation with 1,3-indandione to form the enedione intermediate $[\mathrm{B}]$. Intermediate $[\mathrm{B}]$ then condenses with the condensation product $[\mathrm{A}]$ of amine and ketones to form the intermediate [C] which on cyclization gives the dihydropyridines [D]. Dihydropyridine on subsequent oxidation produces the final product. 


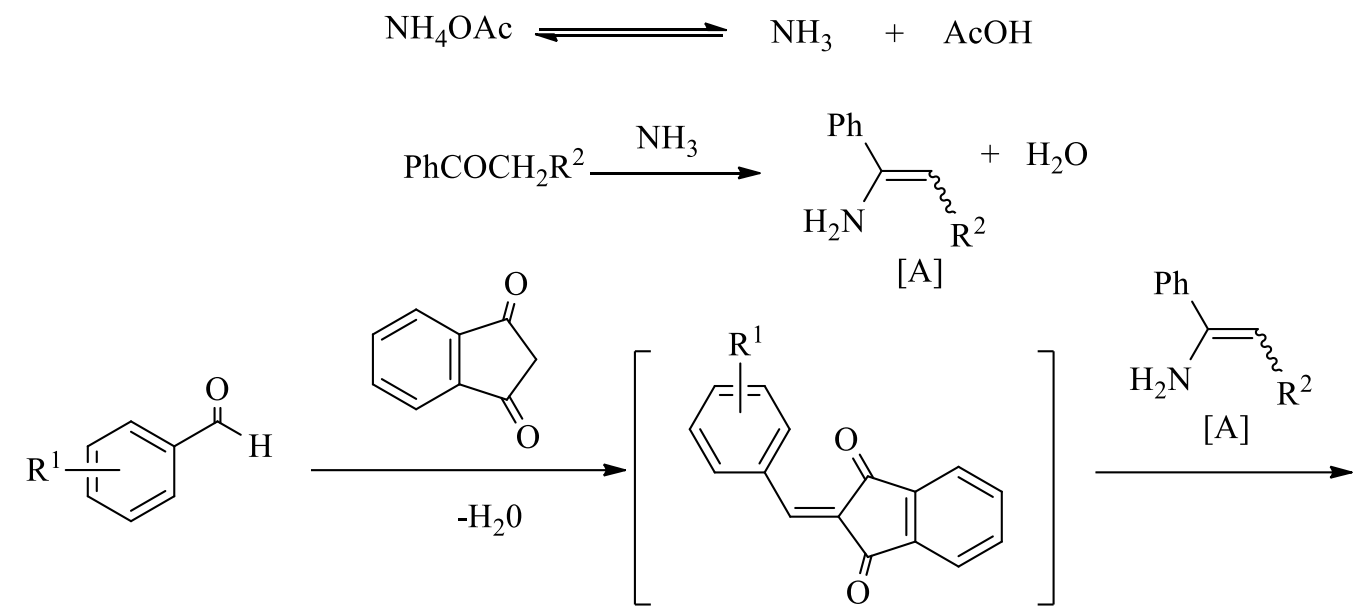

[B]

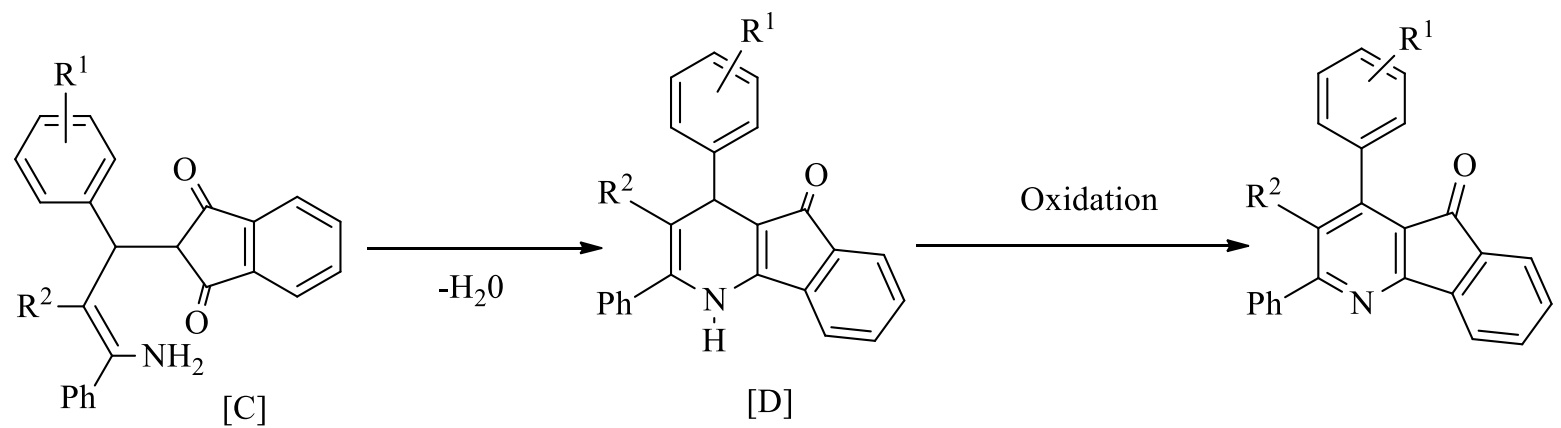

Scheme 2. Probable mechanism for the ceric ammonium nitrate (CAN) catalyzed indeno[1,2b]pyridines synthesis.

In order to establish the scope of this catalytic transformation, the same reaction conditions were applied for the synthesis of 2,3,4,5,6-pentasubstituted pyridines via one-pot threecomponent condensation of aromatic aldehydes $(1 \mathrm{mmol})$, ammonium acetate $(1.3 \mathrm{mmol})$, acetophenone derivatives $(2 \mathrm{mmol})$ as depicted in Scheme 3 and as expected excellent yields were obtained in all cases.

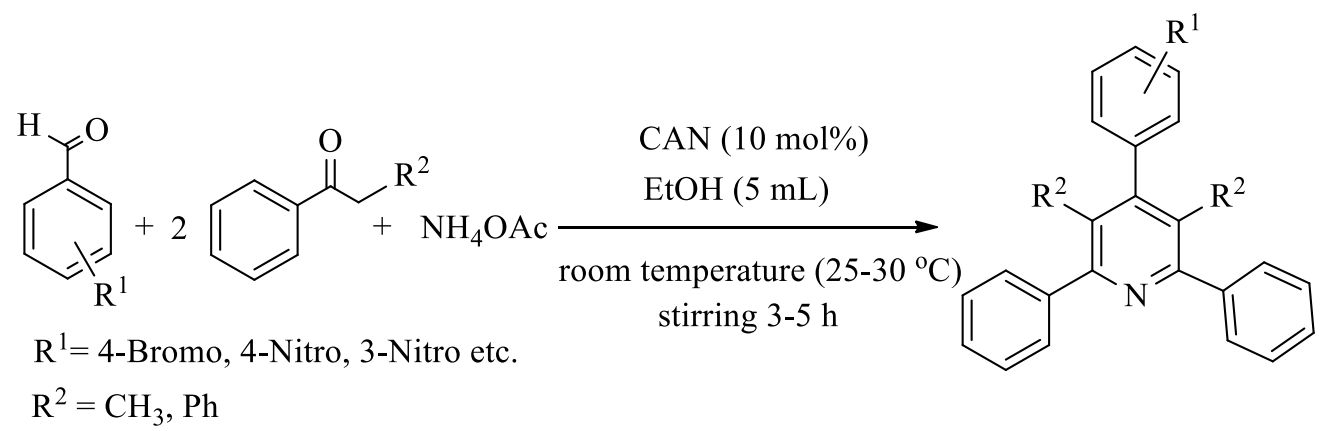

Scheme 3. Synthesis of densely substituted pyridines at room temperature $\left(25-30{ }^{\circ} \mathrm{C}\right)$. 
Table 3. Synthesis of densely substituted pyridines at room temperature $\left(25-30{ }^{\circ} \mathrm{C}\right)$

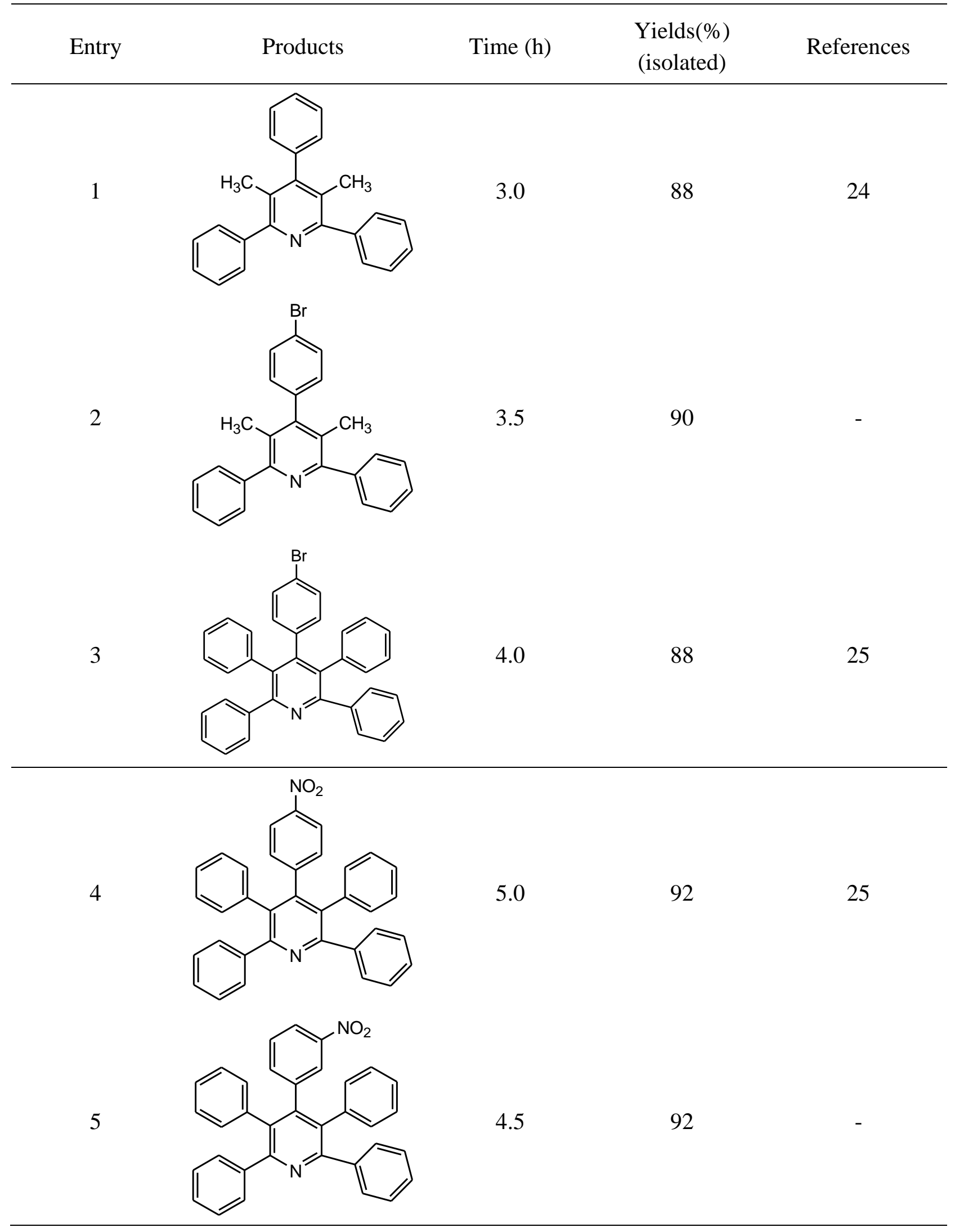




\section{General experimental procedure for indenopyridine formation}

In a $10 \mathrm{~mL}$ round-bottomed flask, aldehyde (1 mmol), 1,3-indandione (1 mmol), propiophenone or 2-phenylacetophenone $(1 \mathrm{mmol})$ and ammonium acetate $(1.3 \mathrm{mmol})$ were stirred in presence of $10 \mathrm{~mol} \%$ of Ceric ammonium nitrate (CAN) in ethanol $(5 \mathrm{~mL})$ at room temperature for the stipulated time (Table 2). The progress of the reaction was monitored by TLC. After completion of the reaction, the reaction mixture was diluted with water $(10 \mathrm{~mL})$ and extracted with ethyl acetate $(3 \times 10 \mathrm{~mL})$. The organic layer was dried over anhydrous $\mathrm{Na}_{2} \mathrm{SO}_{4}$, concentrated and recrystallised from hot ethanol to afford pure product. In case of the 2,3,4,5,6-pentasubstituted pyridines (Scheme 3, Table 3), same procedure was followed except taking 2 mmol of either propiophenone or 2-phenylacetophenone. The IR, ${ }^{1} \mathrm{H}$ NMR and ${ }^{13} \mathrm{C} \mathrm{NMR}$ data of three representative compounds are given below. The spectral data for all the other new compounds are given in the Supplementary Section.

3-Methyl-4-(4-nitrophenyl)-2-phenyl-indeno[1,2-b]pyridin-5-one (Table 2, entry 4). Yellow solid, mp 248-250 ${ }^{\circ} \mathrm{C}(\mathrm{EtOH})$; UV $\left[\lambda_{\max }\left(\mathrm{CH}_{2} \mathrm{Cl}_{2}\right)\right]=381.9 \mathrm{~nm}(\varepsilon=1690)$; IR $(\mathrm{KBr}): 3071,1715$, 1561, 1518 and $1346 \mathrm{~cm}^{-1} .{ }^{1} \mathrm{H}$ NMR $\left(300 \mathrm{MHz} \mathrm{CDCl}_{3}\right) \delta: 8.38(\mathrm{~d}, J=8.4 \mathrm{~Hz}, 2 \mathrm{H}), 7.92(\mathrm{~d}, J=$ $7.2 \mathrm{~Hz}, 1 \mathrm{H}), 7.73-7.42(\mathrm{~m}, 9 \mathrm{H}), 7.40(\mathrm{t}, J=7.5 \mathrm{~Hz}, 1 \mathrm{H}), 2.07(\mathrm{~s}, 3 \mathrm{H}) .{ }^{13} \mathrm{C} \mathrm{NMR}(75 \mathrm{MHz}$, $\left.\mathrm{CDCl}_{3}\right) \delta: 191.0,163.8,162.3,147.9,146.9,142.7,141.9,139.9,135.3,135.0,131.0,129.5$, 129.1, 129.0, 128.9, 128.5, 123.9, 123.7, 123.4, 121.3, 17.6; Anal. Calcd. for $\mathrm{C}_{25} \mathrm{H}_{16} \mathrm{~N}_{2} \mathrm{O}_{3}$; C: 76.52, H: 4.11, N: 7.14\%. Found: C: 76.39, H: 4.21, N: 7.17\%.

2,3-Diphenyl-4-(4-nitrophenyl)-indeno[1,2-b]pyridin-5-one (Table 2, entry 10). Yellow crystalline solid, mp 190-192 ${ }^{\circ} \mathrm{C}(\mathrm{EtOH}) ; \mathrm{UV}\left[\lambda_{\max }\left(\mathrm{CH}_{2} \mathrm{Cl}_{2}\right)\right]=385.5 \mathrm{~nm}(\varepsilon=1687)$; IR $(\mathrm{KBr})$ : 3046, 1714, 1587, 1456, 1176 and $786 \mathrm{~cm}^{-1} .{ }^{1} \mathrm{H}$ NMR $\left(300 \mathrm{MHz}, \mathrm{CDCl}_{3}\right) \delta: 8.10(\mathrm{~d}, J=8.7 \mathrm{~Hz}$, $2 \mathrm{H}), 8.02(\mathrm{~d}, J=7.2 \mathrm{~Hz}, 1 \mathrm{H}), 7.65$ (br. t, $J=7.8 \mathrm{~Hz}, 2 \mathrm{H}), 7.46$ (t, $J=7.5 \mathrm{~Hz}, 1 \mathrm{H}), 7.42-7.35$ (m, 2H), 7.32-7.19 (m, 5H), 7.14-7.00 (m, 3H), $6.38(\mathrm{~d}, J=7.1 \mathrm{~Hz}, 2 \mathrm{H}) ;{ }^{13} \mathrm{C} \mathrm{NMR}\left(75 \mathrm{MHz}, \mathrm{CDCl}_{3}\right)$ $\delta: 190.8,164.3,162.6,147.3,146.0,142.6,141.2,139.7,136.1,135.3,134.8,131.2,131.1$, $130.5,130.0,128.5,128.1,127.8,127.4,123.9,123.0,122.7,121.3$; Anal. Calcd. for $\mathrm{C}_{30} \mathrm{H}_{18} \mathrm{~N}_{2} \mathrm{O}_{3}$; C: 79.28, H: 3.99, N: 6.16\%. Found: C: 79.19, H: 4.06, N: 6.18\%.

4-(4-Bromophenyl)-3,5-dimethyl-2,6-diphenylpyridine (Table 3, entry 2). Pale yellow solid, mp 228-230 ${ }^{\circ} \mathrm{C}(\mathrm{EtOH})$; IR (KBr): 3043, 2989, 1547, 1123 and $750 \mathrm{~cm}^{-1} .{ }^{1} \mathrm{H}$ NMR (300 MHz, $\left.\mathrm{CDCl}_{3}\right) \delta: 7.64(\mathrm{~d}, J=8.3 \mathrm{~Hz}, 2 \mathrm{H}), 7.56(\mathrm{dd}, J=7.6$ and $1.4 \mathrm{~Hz}, 4 \mathrm{H}), 7.42(\mathrm{t}, J=7.2 \mathrm{~Hz}, 4 \mathrm{H})$, $7.36(\mathrm{dd}, J=7.6$ and $1.4 \mathrm{~Hz}, 2 \mathrm{H}), 7.11(\mathrm{~d}, J=8.3 \mathrm{~Hz}, 2 \mathrm{H}), 2.01(\mathrm{~s}, 6 \mathrm{H}) .{ }^{13} \mathrm{C} \mathrm{NMR}(75 \mathrm{MHz}$, $\left.\mathrm{CDCl}_{3}\right) \delta: 156.3,150.5,141.2,138.7,132.2,130.1,129.4,128.1,127.7,127.2$, 121.6, 18.2; Anal. Calcd. for $\mathrm{C}_{25} \mathrm{H}_{20} \mathrm{BrN}$; C: 72.47, H: 4.87, N: 3.38\%. Found: C: 72.36, H: 4.94, N: $3.42 \%$.

\section{Conclusions}

In conclusion, a novel, facile, one-pot, multicomponent methodology for the synthesis of new indeno[1,2- $b$ ]pyridines, a privileged medicinal scaffold catalyzed by $10 \mathrm{~mol} \%$ of ceric ammonium nitrate $(\mathrm{CAN})$ has been developed in high yield at room temperature $\left(25-30{ }^{\circ} \mathrm{C}\right)$. The 
same protocol was also been extended for the rapid construction several 2,3,4,5,6pentasubstituted pyridines at room temperature $\left(25-30{ }^{\circ} \mathrm{C}\right)$. The methodology offers several advantages including mild reaction conditions, easy work-up, clean reaction profile, shorter reaction time, and wide range of substrate applicability that make it a useful and attractive process for the synthesis of substituted pyridine derivatives.

\section{Acknowledgements}

One of the authors (PKT) thanks the University Grants Commission, New Delhi for his fellowship (SRF). We also thank the CAS Instrumentation Facility, Department of Chemistry, University of Calcutta for spectral data. We also acknowledge grant received from UGC funded Major project, F. No. 37-398 / 2009 (SR) dated 11-01-2010.

\section{References and Notes}

1. (a) Dömling, A.; Ugi, I. Angew. Chem., Int. Ed. 2000, 39, 3168. (b) Dömling, A. Chem. Rev. 2006, 106, 17. (c) Zhu, J.; Bienaymé, H. Eds. Multicomponent Reactions; Wiley-VCH: Weinheim, 2005. (d) Armstrong, R. W.; Combs, A. P.; Tempest, P. A.; Brown, S. D.; Keating, T. A. Acc. Chem. Res. 1996, 19, 123.

2. (a) Schreiber, S. L. Science 2000, 287, 1964. (b) Burke, M. D.; Schreiber, S. L. Angew. Chem., Int. Ed. 2004, 43, 46.

3. Zhang, J.; El-Shabrawy, A.-R. O.; El-Shanawany, M. A.; Schiff, P. L.; Slatkin, D. J. J. Nat. Prod. 1987, 50, 800.

4. (a) Heintzelman, G. R.; Averill, K. M.; Dodd, J. H.; Demarest, K. T.; Tang, Y.; Jackson, P. F. PCT Int. Appl. WO 2003088963, 2003. (b) Heintzelman, G. R.; Averill, K. M.; Dodd, J. H.; Demarest, K. T.; Tang, Y.; Jackson, P. F. U.S. Pat. Appl. Publ. US 2004082578, 2004.

5. Safak, C.; Simsek, R.; Altas, Y.; Boydag, S.; Erol, K. Boll. Chim. Farm. 1997, 136, 665.

6. Rentzea, C.; Meyer, N.; Kast, J.; Plath, P.; Koenig, H.; Harreus, Al.; Kardorff, U.; Gerber, M.; Walter, H. Ger. Offen DE 4301426, 1994.

7. Moustafa, A. H.; El-Abbady, S. A.; Gado, S. H.; El-Borai, M. A. Pharmazie 1983, 38, 221.

8. (a) Tadic, D.; Cassels, B. K.; Cave, A.; Goulart, M. P. F.; de Oliveira, A. B. Phytochemistry 1987, 26, 1551. (b) Prostakov, N. S.; Vasil'ev, G. A.; Zvolinskii, V. P.; Varlamov, A. V.; Savina, A. A.; Sorokin, O. I.; Lopatina, N. D. Chem. Heterocycl. Compd. (Engl. Transl.) 1975, 971.

9. Prostakov, N. S.; Soldatenkov, A. T.; Radzhan, P. K.; Fedorov, V. O.; Fomichev, A. A.; Rezakov, V. A. Chem. Heterocycl. Compd. (Engl. Transl.) 1982, 390.

10. Zhang, J.; el-Shabrawy, O.; el-Shabrawy, M. A.; Schiff, P. L., Jr.; Slatkin, D. J. J. Nat. Prod. 1987, 50, 800 . 
11. Padwa, A.; Heidelbaugh, T. M.; Kuethe, J. T. J. Org. Chem. 2000, 65, 2368.

12. Alves, T.; de Oliveira, A. B.; Snieckus, V. Tetrahedron Lett. 1988, 29, 2135.

13. (a) Tu, S.; Jiang, B.; Jia, R.; Zhang, J.; Zhang, Y. Tetrahedron Lett. 2007, 48, 1369. (b) Tu, S.; Jiang, B.; Yao, C.; Jiang, H.; Zhang, J.; Jia, R.; Zhang, Y. Synthesis 2007, 9, 1366.

14. Mukhopadhyay, C.; Tapaswi, P. K.; Butcher, R. J. Tetrahedron Lett. 2010, 51, 1797.

15. (a) Itoh, K.; Akira Horiuchi. C. Tetrahedron 2004, 60, 1671. (b) Han, B.; Jia, X. -D.; Jin, X. -L.; Zhou, Y. -L.; Yang, L.; Liu, Z. -L.; Yu W. Tetrahedron Lett. 2006, 47, 3545.

16. (a) Nair V.; Deepthi, A. Chem. Rev. 2007, 107, 1862. (b) Sridharan V.; Menendez, J. C. Org. Lett. 2008, 10, 4303; (c) Chang, M.- Y.; Wu, T.- C.; Lin C.- Y.; Hung, C.- Y. Tetrahedron Lett. 2006, 47, 8347.

17. Pfister, J. R. Synthesis 1990, 689.

18. Sridharan, V.; Maiti, S.; Menéndez, J. C. Chem. Eur. J. 2009, 15, 4565.

19. Sridharan, V.; Maiti, S.; Menéndez, J. C. J. Org. Chem. 2009, 74, 9365.

20. Chang, M. -Y.; Linb, C. -Y.; Wua, T. -C. Tetrahedron Lett. 2006, 47, 5445.

21. Reddy, C. S.; Raghu, M. Chin. Chem. Lett. 2008, 19, 775.

22. Kidwai, M.; Bhatnagar, D. Tetrahedron Lett. 2010, 51, 2700.

23. (a) Mukhopadhyay, C.; Tapaswi, P. K. Tetrahedron Lett. 2008, 49, 6237. (b) Mukhopadhyay, C.; Tapaswi, P. K.; Drew, M. G. B. Tetrahedron Lett. 2010, 51, 3944. (c) Mukhopadhyay, C.; Tapaswi, P. K.; Butcher, R. J. Org. Biomol. Chem. 2010, 8, 4720.

24. (a) Elshafie, Sayed M. M. J. prakt. Chemie 1982, 324(1), 149. (b) Barluenga, J.; Joglar, J.; Gonzalez, F. J.; Gotor, V.; Fustero, S. J. Org. Chem. 1988, 53, 5960.

25. Jiang, B.; Hao, W.-J.; Wang, X.; Shi, F.; Tu, S.-J. J. Comb. Chem. 2009, 11, 846. 